\title{
Detectability of Fibrils by Dilation Technique in Digital Mammography
}

\section{Siti Shuwaibah Che Omara,*, Wan Muhamad Saridan Wan Hassan ${ }^{a}$, Norehan Mohd Nor ${ }^{a}$, Mohd Syafiq Mohd Suri ${ }^{b}$, Nurul Diyana Shariffc}

a Department of Physics, Faculty of Science, Universiti Teknologi Malaysia, 81310 Skudai, Johor, Malaysia; ${ }^{b}$ School of Mechanical Engineering, Universiti Teknologi Malaysia, 81310 Skudai, Johor, Malaysia; ' Department of Radiology, Hospital Sultanah Aminah, Jalan Persiaran Abu Bakar Sultan, 80100 Johor Bahru, Johor, Malaysia.

Abstract The detectability of fibrils in mammographic phantom images by morphological enhancement was analysed in the present study. Materials that mimic fibrils were imaged by a digital mammography machine at 28 and $29 \mathrm{kV}_{\mathrm{p}}$. The images were processed by a dilation technique to produce second set of images. Receiver operating characteristic analysis was performed to compare the detection performance from the two sets of images. As compared to original images, the $28 \mathrm{kV}$ 's fibrils images from dilation technique become more prominence to be detected by observers. While at $29 \mathrm{kV}$ only a few observers can found the fibrils images from dilation technique. This study suggests morphological enhancement of mammography image did not increase the detection of low frequency signals of the images.

Keywords: Digital Mammography, Receiver Operating Characteristic Analysis, Dilation Technique, Fibrils Performance.

\section{Introduction}

Nowadays, women concerns about breast cancer issues. Breast cancer is like a nightmare for women if they talk about it because it is famous in the female population around the world. Therefore, many women now will be take an action in routinely screening their breast to detect and make a treatment of breast cancer to reduce mortality. Mortality due to breast cancer has increased overall ages in the past decade [1]. Therefore, mortality reduction had been achieved by mammography screening technique and thus, improving the treatment of breast cancer. The most effective tool that almost used to detect the breast cancer in an early stage is using mammography [2].

*For correspondence: sshuwaibah2@graduate. utm.my

Received: 18 Jan 2021

Accepted: 15 August 2021

(C) Copyright Omar. This article is distributed under the terms of the Creative Commons Attribution License, which permits unrestricted use and redistribution provided that the original author and source are credited.
The importance of mammographic screening technique has studied by the previous study. Health Insurance Plan (HIP) of Greater New York reported in December of 1963, women with average aged between 40 to 64 years reduced $25 \%$ of mortality in breast cancer who's received screening mammography [3]. According to Soojin et al. [4], women aged 40 years and older domized amount over 162,981 could reduce mortality from breast cancer if they determine the extant mass screening with single-view mammography. The Swedish National Board of Health and Welfareran proved this amount. Other randomized trials have confirmed there decrease in breast cancer mortality related to mammography with the same range. Second reported by Lim and Halimah [5] in the Malaysian National Cancer Registry in 2004 that 46.2 in 100,000 population was diagnosed with breast cancer in 2003 compared to 130 in 100,000 population in the United States [6]. The only percentage of $65 \%$ women over age 40 years reported was a rapid increase in screening mammography within the prior 2 years start over the year 2015. However, this technique was often small and non-symptomatic on detection of 
breast cancer. Furthermore, it was limited to image processing and 3D imaging application [7]. Therefore, to improved radiological breast imaging techniques, the mammography screening was replaced by digital mammography to have bring about in more accurate detection and characterisation of breast wounds at screening mammography $[8,9]$ Digital mammography produces consequently in higher sensitivity and specificity of breast cancer diagnosis [9]. In addition, digital mammography was used easily in image processing and 3D imaging application [7]. It also exposes the breast to much less radiation compared to chest X-ray or X-rays of extremity. Thus, the digital mammography technique was used in the present study.

In recent years, there are many researchers proposed algorithm with a set of contrast enhancement function of mammograms. Dhawan et al. [10] proposed the set of contrast to enhance the mammographic features by optimal adaptive neighbourhood image processing. The method can be improved by the enhancing desired but it has barely unseen a little enhancement of noise and background variations. Tomklav Stojic et al. in two different research [11, 12] proposed a new algorithm for local contrast enhancement in digital mammography by using mathematical morphology. The mathematical morphology applied the gray-scale image processing and not efficient in enhancing the micro calcifications in digital mammograms. Another one is enhancement of microcalcifications in digitized mammograms that proposed the multifractal approach. Multifractal was related to the normal state of human tissue to characterize high levels of self-similarity. Aderonke et al. proposed the enhancement of mammograms that can be achieve by increasing the contrast of mammograms at low dose X-ray or by sharpening image edges and boundaries. Its aim is to eliminate the background noise and improve the quality to determine the region of interests (ROIs) in MATLAB [13, 14]. Moradmand [8] proposed different enhancement methods and determine the appropriate one for mammogram enhancement and microcalcification detection. All the mentions literature related to morphological enhancement. Image enhancement is the basic step in image processing. The purpose of image enhancement helps to produce the better and quality image. In this paper, one of the image enhancement use is morphological enhancement.

Receiver Operating Characteristic (ROC) analysis is a one method to evaluates the sensitivity and specificity by calculating the areas under the curves [15]. As the function to determine the sensitivity and specificity, the accuracy of an imaging system can be find using this ROC. Sensitivity or also call true positive fraction (TPF) defined that the probability of disease can identifying in real presenting. While specificity or true negative fraction (TNF) is show the absence of disease when absent [16]. This both related function is call ROC curve. Recently, the application of ROC was extensively welcomed as a tool in expressing uncertainly when making visual judgments about radiological images [17]. Therefore, it is necessary to analyse the detectability of breast cancer image in early stages. To improve the detectability of breast cancer in early stages, a sample of fibrils was used in the present study because the image characteristic of fibrils is mimic to image characteristics of breast cancer tumour. Therefore, the objective of the present study was to analyse the image of fibrils by morphological enhancement specific in dilation technique using digital mammographic and analysed using ROC methodology

\section{Materials and methods}

The mammographic images scored based on subjective interpretation by four observers. The observers are choose among the expert professional such as medical practice and radiologist. They are have the expertise in image processing in x-ray exposure. The images were scored on a scale range from 1 (definitely not present), 2 (probably not present), 3 (no decision possible), 4 (probably present) and 5 (definitely present) [7]. In this study, the discussion is about the morphological enhancement using dilation technique in MATLAB 7.0 software and then the graph is plotting using Receiver Operating Characteristic (ROC) analysis using ROCFIT and CORROC2 [18-20]. ROCFIT is a fits maximum likelihood procedure for ROC models assuming a binormal distribution of the latent variable. While purposes of CORROC2 is to evaluate the maximum possibility approximate the parameters for correlated ROC rating scale data based on an effective partner of the basic bivariate-normal for division result variable. Second applicable of CORROC2 is to calculate the numerical importance of the dissimilarity between the two estimated ROC curves using a univariate z-score test of the difference 
between the areas under the two ROC curves as well as null hypothesis say that the data sets emerge from ROC curves with same area under it. Differences among two ROC curve areas were considered significant in two-tailed hypothesis tests at P-level< 0.05 [21].

\section{Image Acquisition}

In this study, two sets of samples were used as an object. Each set was exposed at different X-rays energy, one was exposed at $28 \mathrm{kV}_{\mathrm{P}}$ and the other one was exposed at $29 \mathrm{kV}$. Every set consist of ten different templates were created using paper sheets that mimic three types of lesions namely nodules, fibrils and micronodules. Each template consists of nine partitions fields of approximately equal size in which the lesions were fixed at different arrangement inside the partition. The images of the samples for both sets were acquired using a Hologic Lorad Selenia digital mammographic machine at the Hospital Sultanah Aminah, Johor Bahru. A "Rachel" Anthropomorphic Breast Phantom Model 169 perspex slab was used as the phantom. Figure 1 shows the setup of the image acquisition. Figure 2 shows the materials used to mimic the nodules, fibrils and micro nodules were simulated with candle wax, folded aluminium foil, optic fibre, nylon and mustard, respectively. These papers are focusing on fibrils materials which are nylon and optic fibre image only. Figure 3 shows the one of the templates of original image, which is directly, expose to X-rays energy using mammogram machine. Meanwhile, Figure 4 was the same image of Figure 3 but it has through the morphological enhancement processed to improve the quality image and its name as the dilated image. The resulting images were then processed by morphological enhancement using dilation technique that was described in subsection morphological enhancement technique. Next subsection will cover the Receiver Operating Characteristic (ROC) analysis that use to get the result of set of original and dilated image.

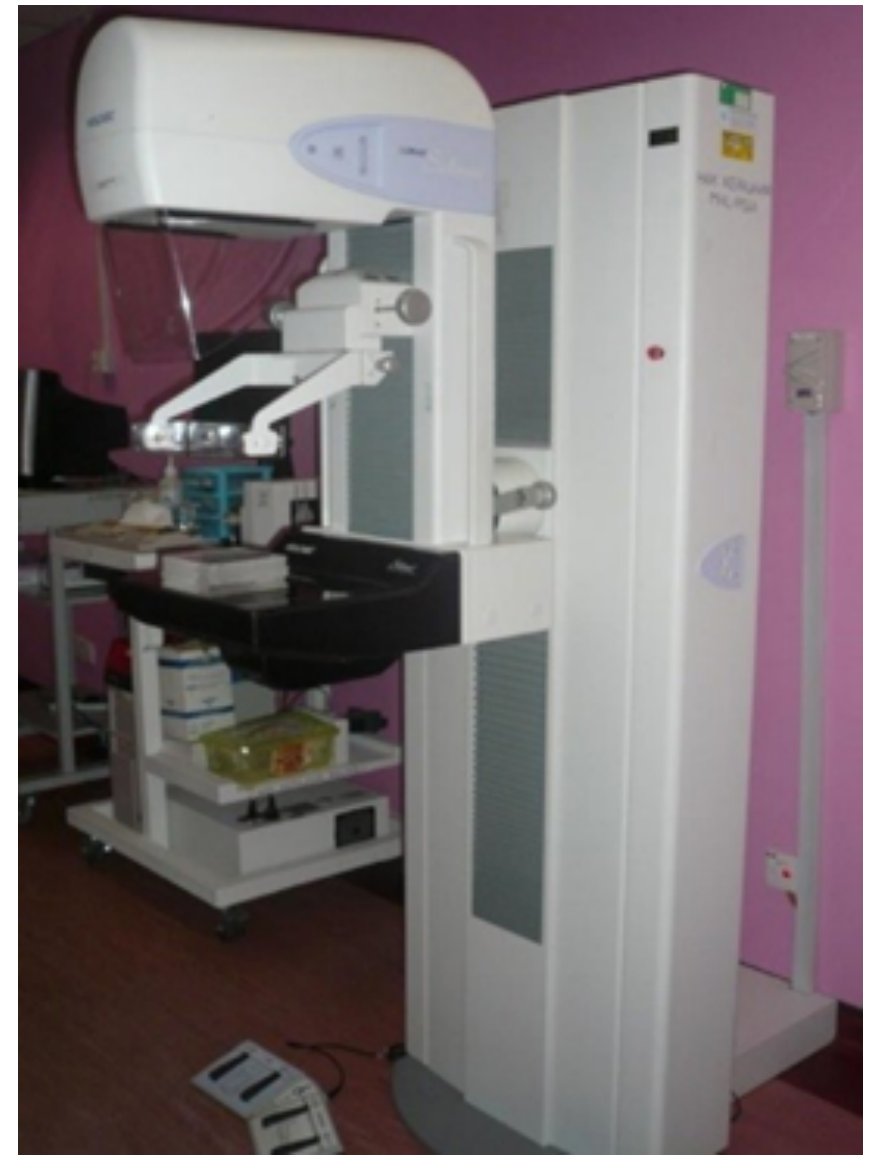

Figure 1. Setup of image acquisition 


\section{MJFAS}

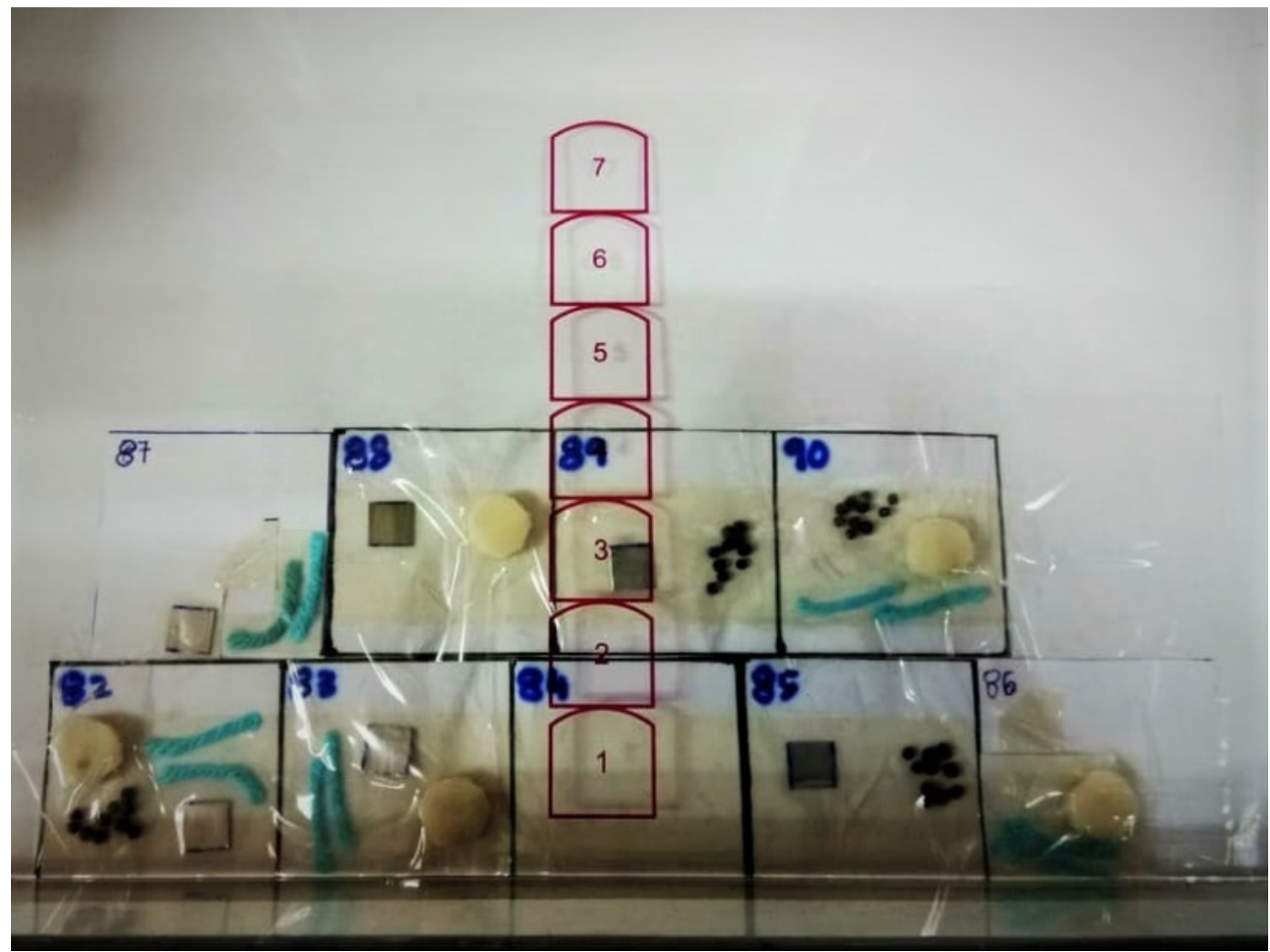

Figure 2. Materials used to mimic the nodules, fibrils and micronodules

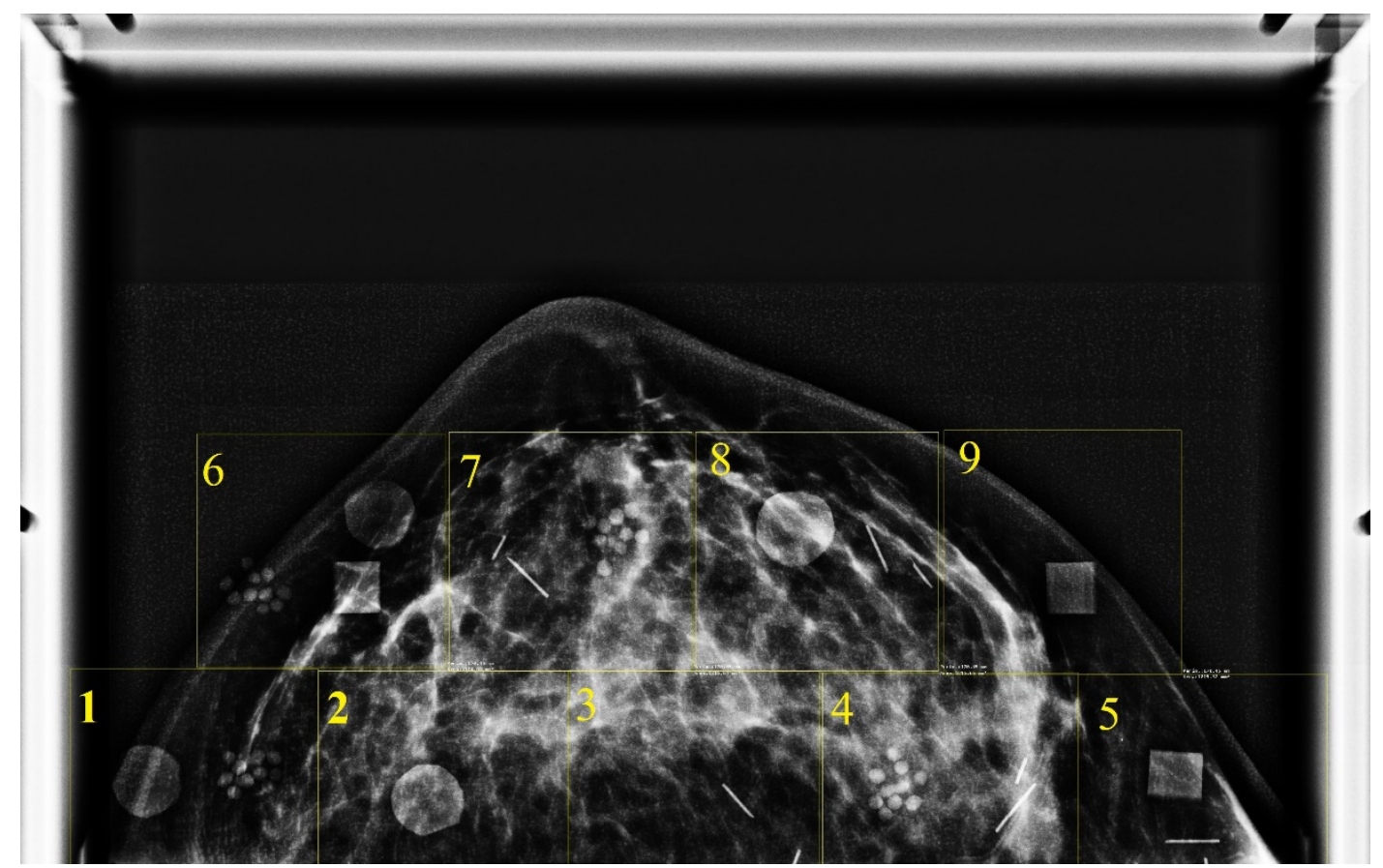

Figure 3. Original image. 


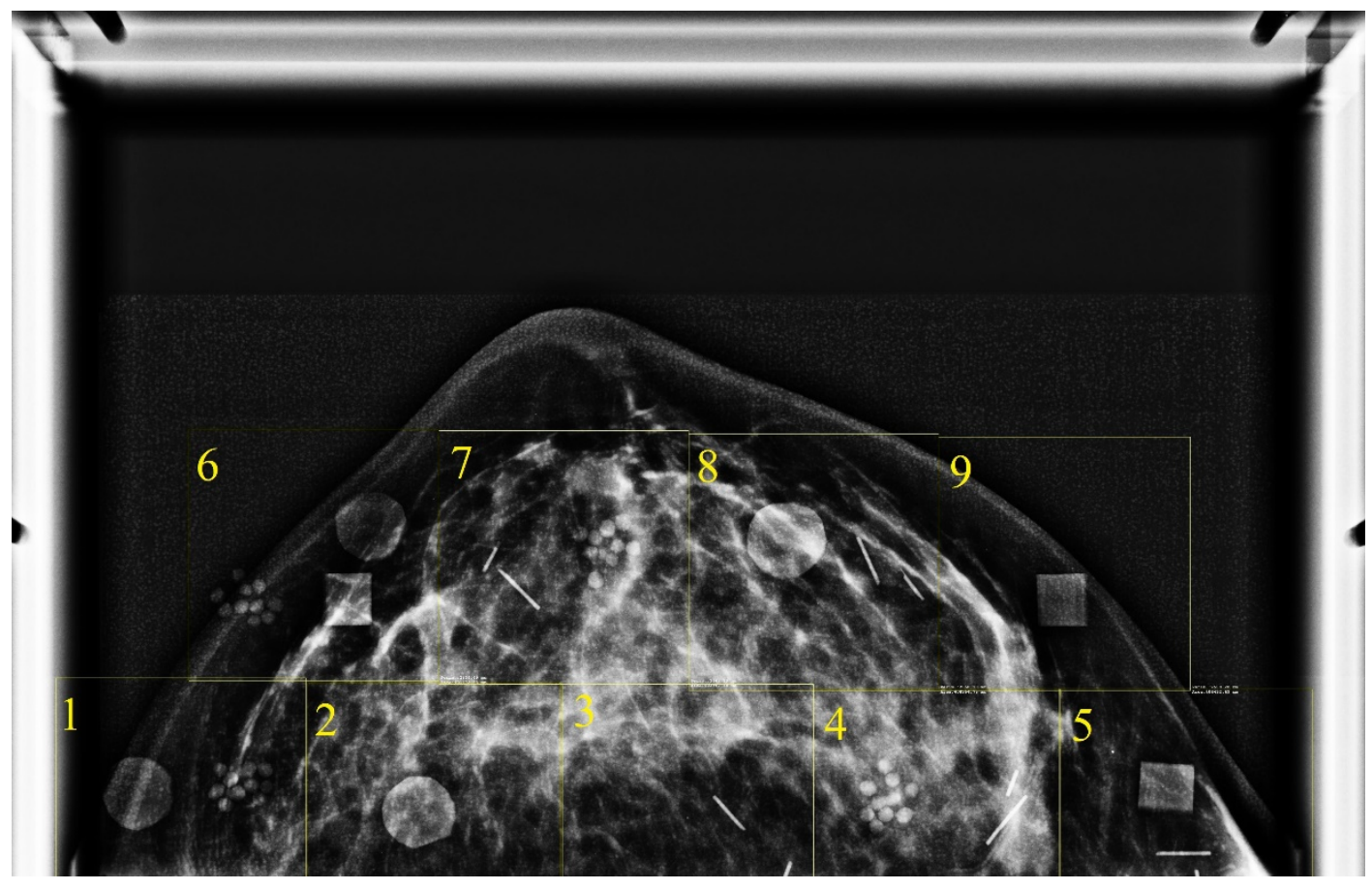

Figure 4. Dilated image

\section{Morphological Enhancement Technique}

In the morphological enhancement technique, there are many methods to enhance the image. One of the method is using dilation technique. Dilation is the procedure of enlarging or thickening an object in a binary image. The shape mentioned to as a structuring element handles the typical way and the extent of this thickening. Mathematically, dilation defined in term of set operations, which is dilation of $A$ by $B$, denoted $A \oplus B$, and is defined as

$$
A \oplus B=\{z \mid(\widehat{B}) z \cap A \neq \emptyset\}
$$

where $\varnothing$ is the unfilled set and $B$ is known as the structuring element. According to Gonzalez et al. [22], the dilation of $A$ and $B$ is the set consisting of all the structuring element originations where the reflected and translated $B$ overlaps at least some portion of $A$. In this paper, the dilation of commutative that is $A \oplus B=B \oplus A$. It is a convention in image processing to let the first operand of $A \oplus B$ be the image and the second operand be the structuring element, which usually is much smaller than the image. After that, the mammographic phantom images were enhanced using image processing toolbox (IPT) function strel. Strel constructs structuring elements with a variety of shapes and size. Its basic syntax is

$$
\text { se }=\text { strel (shape, parameters) }
$$

The strel is useful in flat structuring element which support arbitrary shapes which is a string that determines the desired shape and parameters is a list of parameters that specify the information about the shape, such as its size [22, 23]. The used of strel ('disk', 5) to returns a disk-shape structuring element that extends \pm 5 pixels along the horizontal and vertical axes. In the enhancement, the images were dilated using a disk shaped structuring element to reduce noise and improve image contrast. Lastly, the morphological closing was applied to the image after dilation [23].

The dilation technique was applied to increase the original image. For instance, the dilation was performed the IPT function 'imdilate' as shown in Equation (3): 


$$
A 2 \text { = imdilate }(A, B)
$$

where $A$ are original image and $A 2$ are enhance images using dilation technique, and $B$ is a matrix of 0 s and $1 \mathrm{~s}$ that specifies the image structuring element. The Equation (3) was structured in matrix form, like:

$\begin{array}{lll}0 & 1 & 0 \\ 1 & 1 & 1 \\ 0 & 1 & 0\end{array}$

Using MATLAB software, the commands read the image from the file, form the image structuring element matrix to perform the dilation image, and display the result as image enhancement. The process of naming the command was repeated many times with a different image until the set of images was finish. After finishing the entire image processing, the score of the image was made by a few observers as mention before and the result of making the score was analysed using the ROC curve.

\section{Receiver Operating Characateristic (ROC) Analysis}

ROC analysis usually performed to explore the characteristics and accuracy of detecting the 2 sets of different images depending on subjective interpretation [24, 25]. CORROC2 and ROCFIT tools are used to process the cluster data from the ROC score calculation data sets and operating points [20, 26, 27].

In the input of the CORROC2, the cases of positive and negative is need to note as the confidence rating scale from the ROC data sets. From that, the CORROC2 will considered the difference of two proximate ROC curves from the two conditions namely the original image and enhanced image using morphological techniques. The system in the computer software will process the true positive traction (TPF) value for original images that label as condition $X$ and false positive fraction (FPF) value is label as condition $Y$ for enhancement images. After running the software, the area under the fitted ROC curves appears from the two states with standard deviation estimates and standard correlation [20, 28]. Meanwhile, the ROCFIT is only suitable for one diagnostic test to determine the broad area index of ROC $\left(A_{z}\right)$ and standard deviation in binormal ROC curve [27].

\section{Results and discussion}

Figure 5 shows the ROC curves for observer 1 in detecting the fibrils in the original and dilated images at (a) $28 \mathrm{kV}_{\mathrm{P}}$ and (b) $29 \mathrm{kV}_{\mathrm{P}}$ using digital mammographic machine. The detection performance is in the following order (highest to lowest): optical fibre in both energies for original and dilated image have the same value of 1.0000 for areas under the curve (AUC) of the ROC $\left(A_{z}\right)$. The value of AUC for original nylon fibre and dilated nylon fibre image at the energy of $28 \mathrm{kV}_{\mathrm{P}}$ is 0.9879 and 0.9556 , respectively, while at the energy of $29 \mathrm{kV}_{\mathrm{P}}$ is 0.9869 and 0.9647 , respectively. The AUC is a combination of measures on sensitivity (TPF) and specificity (FPF). AUC also measure the overall performance of diagnostic test and it is interpreted as the average value of sensitivity for all possible values of specificity [25, 29]. For dilated nylon fibre, there is a decrease in the value of AUC, which about 0.0323 and 0.0222 reduction from its original image AUC value for $28 \mathrm{kV}_{\mathrm{P}}$ and $29 \mathrm{kV}_{\mathrm{P}}$, respectively. This indicate that the mammography image become worsen after its going through an image processing using dilated method. On the contrary, the value of AUC for optical fibre image remain unchanged before and after image processing. The closer the value of AUC to one, the better the overall diagnostic performance of the test. The lower limit for AUC of the diagnostic test is 0.5 and it is perfectly accurate if the AUC value is equal to one. From the observed AUC value, it found that the optical fibres have a high contrast and frequency compare to nylon fibres. According to [30], the value of $A_{z}$ still can be categorized as excellent test, even though an average test was obtained is $90 \%$ abnormal result. The same procedure done to optical fibre images and the result for both images is maintaining the same values of 1.0000 , which is $100 \%$ perfect test. 
(a)

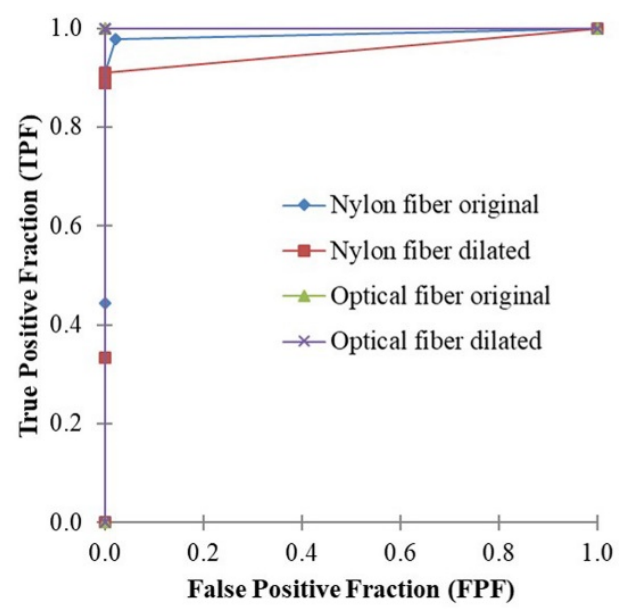

(b)

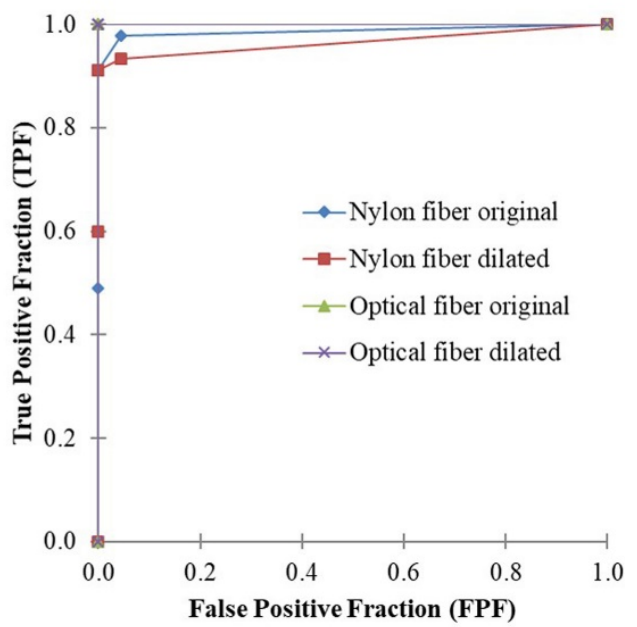

Figure 5. ROC curves of fibrils in original and dilated images at (a) $28 \mathrm{kVp}$ and (b) $29 \mathrm{kVp}$ of the digital mammography machine for observer 1.

Figure 6 illustrate the detection of fibrils at (a) $28 \mathrm{kV}$ and (b) $29 \mathrm{kV}$ mammography images by observer 2. For energy of $28 \mathrm{kV}$, both original and dilated images for optical fibre have the same, the highest detection performance followed by dilated, and the original image of nylon fibre with corresponds to AUC value of $1.0000,0.9886$ and 0.9422 , respectively. Both detection performance of optical fibre are close to upper left corner which is at coordinate $(x=0, y=1)$. It shows that, this images have greater in sensitivity (no false negatives) and specificity (no false positives). While, for the nylon fibre, the dilated image shows increasing in AUC value compare to its original image with percentage of $4.92 \%$. That means the curve shows the results of a less sensitive and specific test. A curves in this Figure 6 (a) are still in the correctly predictive result because the values plotted above and to the left of the line of equality or the random chance line [29]. From Figure $6(b)$, the AUC of ROC values for energy of $29 \mathrm{kV}$ are $1.0000,0.9889,0.9706$ and 0.8822 with respect to dilated optical fibre image, original optical fibre image, original nylon fibre image and dilated nylon fibre image (using the following order highest to lowest). Even though the value of AUC for dilate nylon fibre image have a value of 0.8822 , it is noted as a good test because the prediction model would be considered strong when $\geq 0.8$ [29].

An ROC curve of TPF (sensitivity) as a function of FPF (1- specificity) that score by observer 3 is shown in Figure 7. This figure shows the areas under the ROC curve for nylon fibre and optical fibre images that exposed to energy of at (a) $28 \mathrm{kV}_{\mathrm{P}}$ and (b) $29 \mathrm{kV}$. For both energy of $28 \mathrm{kV} \mathrm{P}_{\mathrm{P}}$ and $29 \mathrm{kV}$, the dilated optical fibre image have a better value of areas under the ROC curve than original optical fibre image with increasing about 0.0111 from 0.9889 to 1.0000 . Based on this result, both detection performance of optical fibres images are in the correctly predictive result. Inversely to optical fibre image, the original nylon fibre image has a high value of $A_{z}$ compare to dilated nylon fibre image with different value of 0.0136 which is from 0.9632 to 0.9496 . Its shows that the result of the score is depend on the individually. At the same procedure, the observer 3 marked the second image score called dilated image after marked the original image score. The resulting score from observer 3 for both images illustrated in Figure 7 (b). Figure 7 (b) have a slightly the same pattern with observer 2 (Figure $6(b)$ ) which is the following order of AUC value from highest to lowest is dilated optical fibre image, original optical fibre image, original nylon fibre image and dilated nylon fibre image and their AUC of the ROC are 1.0000, 0.9889, 0.9632 and 0.9356 , respectively. All fours of the images considered strong with value of $A U C \geq 0.8$, which categorized as excellent and perfect test. 
Figure 8 shows the areas under the ROC curve (AUC) of fibrils for observer 4 for mammography images at (a) $28 \mathrm{kV}_{\mathrm{P}}$ and (b) $29 \mathrm{kV}$. The plots of $A_{z}$ in Figure 8 (a) shows the value of AUC for dilated nylon fibre image is increase by 0.0037 from original nylon fibre image $\left(A_{z}=0.9889\right)$ to 0.9926 correspond to its performance detection. Figure 8 (b) shows the dilated nylon fibre image also have a better $A_{z}$ compare to original nylon fibre image which is 0.9701 and 0.9667 , respectively. The interpretation of this $A_{z}$ values is an excellent test. While for both energy, all the optical fibre images maintained at 1.0000 for the AUC value. It is indicate that this is the perfect test for observer 4. Overall from all the graphs (Figure 5-8), the resulting detection performance curve from observer 1 and 4 are in a good shape compared to observer 2 and 3 . This is due to the effect of utilizing different types of observer as respondents. Factor such as gender, age and occupation whether their work involved in evaluating an image or not can affected the results.

(a)

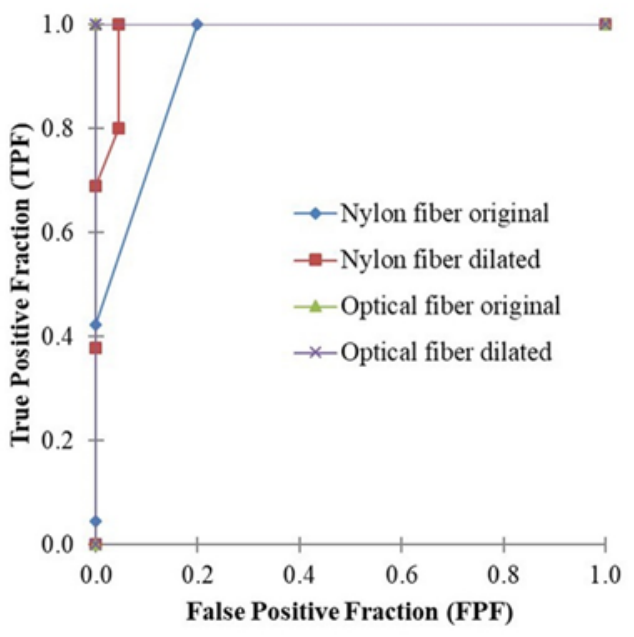

(b)

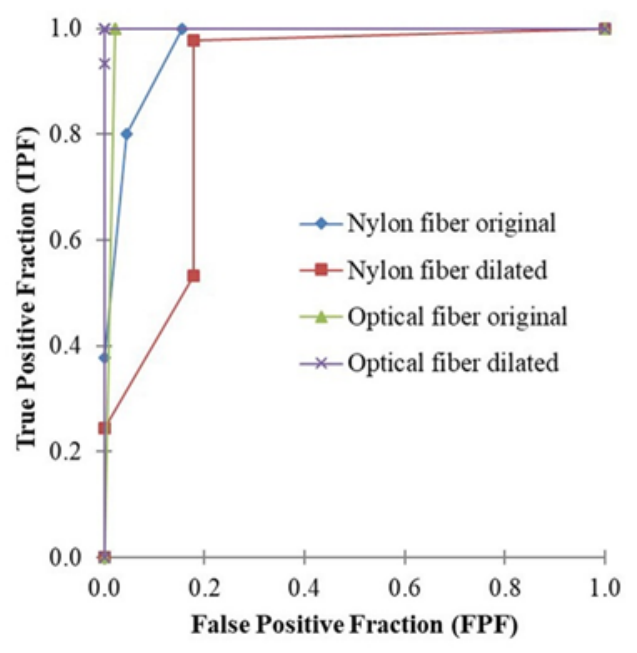

Figure 6. ROC curves of fibrils in original and dilated images at (a) $28 \mathrm{kVp}$ and (b) $29 \mathrm{kVp}$ of the digital mammography machine for observer 2.

(a)

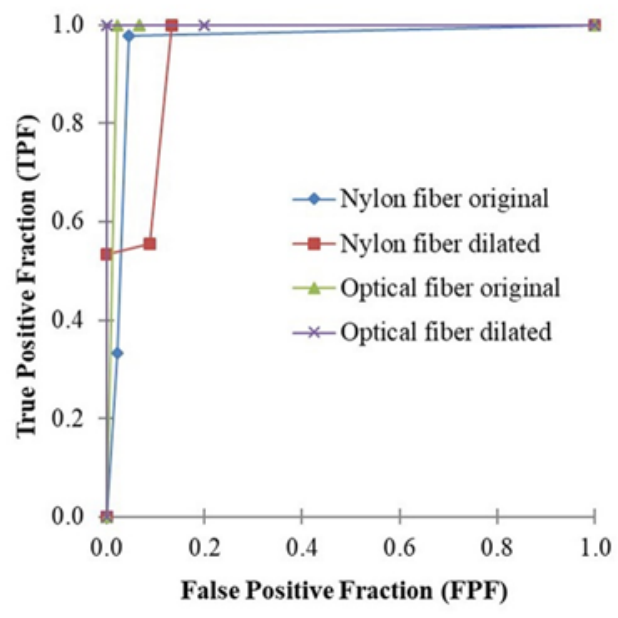

(b)

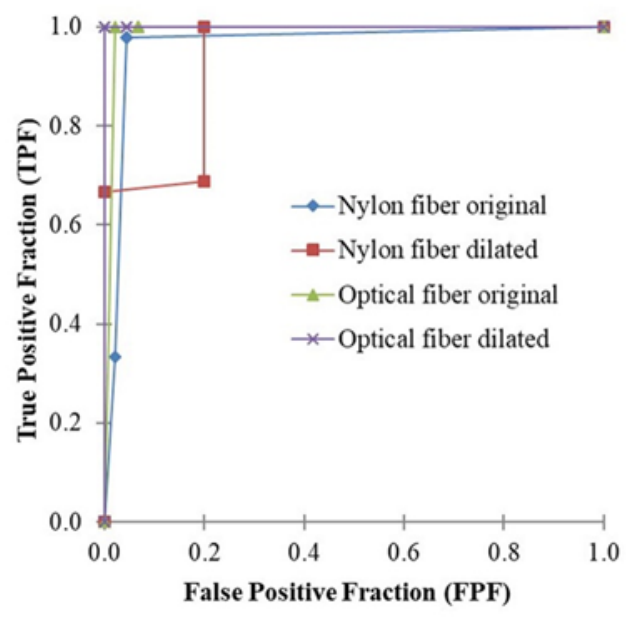

Figure 7. ROC curves of fibrils in original and dilated images at (a) $28 \mathrm{kVP}$ and (b) $29 \mathrm{kVP}$ of the digital mammography machine for observer 3. 
(a)

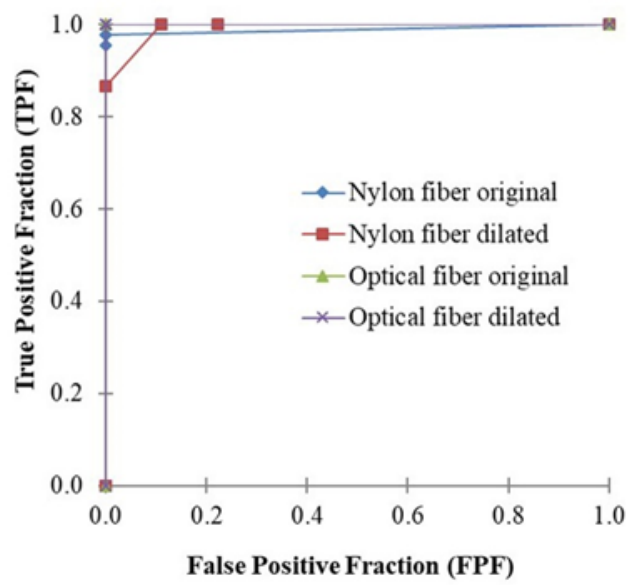

(b)

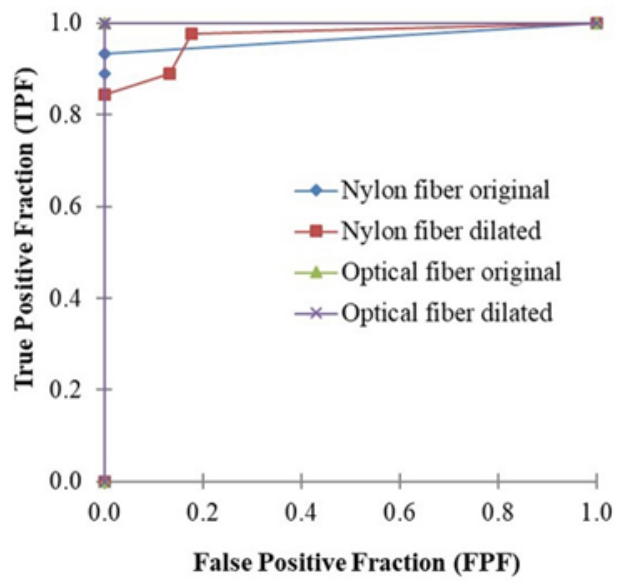

Figure 8. ROC curves of fibrils in original and dilated images at (a) $28 \mathrm{kVp}$ and (b) $29 \mathrm{kVp}$ of the digital mammography machine for observer 4.

Table 1 shows the AUC value of ROC $\left(A_{z}\right)$ for the four observers in detecting the original and dilated images of fibrils at $28 \mathrm{kV}$ and $29 \mathrm{kV}$ of the digital mammographic machine. The $A_{z}$ for original and dilated optical fibre images for all observers are the same AUC value which is 1.0000 except observer 2 (energy at $29 \mathrm{kV}$ ) and observer 3 (both energies) have a value of 0.9889 in optical fibre original image. It shows that, the value of AUC increased for two observers when using image from dilation technique. Sightly different to nylon fibre at energy of $28 \mathrm{kV}$, there are two observers show the increasing value in original image to dilated image which is from 0.9422 to 0.9886 for observer 2 and from 0.9889 to 0.9926 for observer 4. For the other two observers, their AUC value decreasing from original image to dilated image with value of 0.9879 to 0.9556 and 0.9632 to 0.9496 for observer 1 and 3 , respectively. While for energy of $29 \mathrm{kV}$, both result from observer 2 \& 3 shows increasing in AUC value from optical fibre original image to dilated image (0.9889 to 1.0000$)$. The other two observers (observer 1 and 4 ) have a same value of $A_{z}$ which is 1.0000 . For nylon fibre, three observers downgrade the image performance from original to dilated image in the following order: ( 0.9869 to $0.9647,0.9706$ to 0.8822 and 0.9632 to 0.9356 ) for observer 1,2 and 3, respectively. Only observer 4 indicates that dilated technique have improve the detection performance of images. This is based on observed value of AUC for observer 4 which have shown significant increment from original nylon fibre image to dilated nylon fibre image. It can be say that the nylon fibre is a difficult material to detect the contrast and frequency at energy of $28 \mathrm{kV}_{\mathrm{P}}$ and $29 \mathrm{kV}$. As compared to optical fibre images, the existence of nylon fibre is difficult to detect. Moreover, the utilizing of dilated technique still cannot improved the detection performance of the nylon fibre image.

Table 1. Observer performance by areas under the ROC curve in detection of fibrils in original and dilated images at energy at $28 \mathrm{kVp}$ and $29 \mathrm{kVp}$ of the digital mammographic machine.

\begin{tabular}{ccccccccc}
\hline Observers & \multicolumn{2}{c}{$\begin{array}{c}\text { Nylon Fibre Original } \\
\text { Image }\end{array}$} & \multicolumn{2}{c}{$\begin{array}{c}\text { Nylon Fibre Dilated } \\
\text { Image }\end{array}$} & \multicolumn{2}{c}{$\begin{array}{c}\text { Optical Fibre } \\
\text { Original Image }\end{array}$} & Optical Fibre Dilated Image \\
\hline & $28 \mathrm{kVp}$ & $29 \mathrm{kVp}$ & $28 \mathrm{kVp}$ & $29 \mathrm{kVp}$ & $28 \mathrm{kVp}$ & $29 \mathrm{kVp}$ & $28 \mathrm{kVp}$ & $29 \mathrm{kVp}$ \\
\hline 1 & 0.9879 & 0.9869 & 0.9556 & 0.9647 & 1.0000 & 1.0000 & 1.0000 & 1.0000 \\
2 & 0.9422 & 0.9706 & 0.9886 & 0.8822 & 1.0000 & 0.9889 & 1.0000 & 1.0000 \\
3 & 0.9632 & 0.9632 & 0.9496 & 0.9356 & 0.9889 & 0.9889 & 1.0000 & 1.0000 \\
4 & 0.9889 & 0.9667 & 0.9926 & 0.9701 & 1.0000 & 1.0000 & 1.0000 & 1.0000 \\
\hline
\end{tabular}




\section{Conclusions}

The morphological enhancement by dilation technique of digital mammographic images was studied by ROC analysis. Original images and morphological enhanced images using dilation technique were prepared. The result shows that the dilation techniques did not increase the detection of fibrils for at both 28 and $29 \mathrm{kV}_{\mathrm{p}}$. The study indicates that the morphological enhancement by dilation technique is not a good image processing procedure to increase the detection of low frequency signals in mammographic images. For further study, is recommended to expose the new energy range and processes the images using the combination technique of dilation and erosion to enhance the image.

\section{Conflicts of interest}

This paper has a conflict in term of finding observer where is need the radiologist to involve to score the subjective image processing and they have to take a long time to get the result. Although the result does not involve the patient at the hospital, it has involved the machine equipment to expose the sample template.

\section{Acknowledgments}

The authors would like to acknowledge the MOHE HIR Grants number Universiti Teknologi Malaysia for providing financial assistance through FRGS [R.J130000.7817.4F501].

\section{References}

[1] S. Buseman, J. Mouchawar, N. Calonge, and T. Byers, "Mammography screening matters for young women with breast carcinoma: Evidence of downstaging among 42-49-year-old women with a history of previous mammography screening," Cancer, vol. 97, no. 2, pp. 352-358, 2003.

[2] E.A. Sickles, "Breast cancer screening outcomes in women ages 40-49: clinical experience with service screening using modern mammography," J Natl Cancer Inst Monogr, no. 22, pp. 99-104, 1997.

[3] E.D. Pisano, R.E. Hendrick, M.J. Yaffe, et al., "Diagnostic Accuracy of Digital versus Film Mammography: Exploratory Analysis of Selected Population Subgroups in DMIST," Radiology, vol. 246, no. 2, pp. 376-383, 2008.

[4] S. Ahn, M. Wooster, C. Valente, et al., "Impact of Screening Mammography on Treatment in Women Diagnosed with Breast Cancer," Annals of Surgical Oncology, vol. 25, no. 10, pp. 1-8, 2018.

[5] G.L.C. Chye and H. Yahaya, "Second Report of the National Cancer Registry. Cancer Incidence in Malaysia 2003," National Cancer Registry, pp. 1-141, 2004.

[6] N.A. Taib, C.H. Yip, M. Ibrahim, C.J. Ng, and H. Farizah, "Breast Cancer in Malaysia: Are Our Women Getting The Right Message? 10 Year-Experience in A Single Institution In Malaysia," Asian Pacific Journal of Cancer Prevention, vol. 8, no. 1, pp. 141-145, 2007.

[7] A.D.A. Maidment, "Digital mammography," Seminars in Roentgenology, vol. 38, no. 3, pp. 216230, 2003.

[8] H. Moradmand, S. Setayeshi, A.R. Karimian, M. Sirous, and M.E. Akbari, "Comparing the performance of image enhancement methods to detect microcalcification clusters in digital mammography," Iranian Journal of Cancer Prevention, vol. 5, no. 2, pp. 61-68, 2012.

[9] J.R.C. Lameijer, A.M.P. Coolen, A.C. Voogd, et al., "Correction to: Frequency and characteristics of contralateral breast abnormalities following recall at screening mammography," European Radiology, vol. 28, pp. 4205-4214, 2018.

[10] A.P. Dhawan, G. Buelloni, and R. Gordon, "Enhancement of Mammographic Features by Optimal Adaptive Neighborhood," IEEE Transactions on Medical Imaging, vol. MI, no. 1, pp. 8-15, 1986.

[11] T. Stojic and B. Reljin, "Enhancement of Microcalcifications in Digitized Mammograms: Multifractal and Mathematical Morphology Approach," FME Transactions, vol. 38, no. 1, pp. 1-9, 2010.

[12] T. Stojić, I. Reljin, and B. Reljin, "Local Contrast Enhancement in Digital Mammography by using Mathematical Morphology," ISSCS 2005: International Symposium on Signals, Circuits and Systems - Proceedings, vol. 2, pp. 609-612, 2005. 
[13] Y. Kimori, "Mathematical morphology-based approach to the enhancement of morphological features in medical images," Journal of Clinical Bioinformatics, vol. 1, no. 1, p. 33, 2011.

[14] A. Kayode, B. Afolabi, and B. Ibitoye, "An Explorative Survey of Image Enhancement Techniques Used in Mammography," International Journal of Computer Science Issues (IJCSI), vol. 12, no. 1, p. 72, 2015.

[15] C.E. Metz, "ROC methodology in radiologic imaging.," Investigative radiology, vol. 21, no. 9, pp. 720-33, 1986.

[16] N.A. Obuchowski, "Fundamentals of clinical research for radiologists - ROC analysis," American Journal of Roentgenology, vol. 184, pp. 364-372, 2005.

[17] L.E. Bantis and Z. Feng, "Comparison of two correlated ROC curves at a given specificity or sensitivity level," Statistics in Medicine, vol. 35, no. 24, pp. 4352-4367, 2016.

[18] X. Zhai, D.P. Chakraborty, and X. Zhai, "A bivariate contaminated binormal model for robust fitting of proper ROC curves to a pair of correlated, possibly degenerate, ROC datasets," Medical Physics, vol. 44, no. 6, pp. 2207-2222, 2017.

[19] C.E. Metz, "ROC analysis in medical imaging: a tutorial review of the literature.," Radiological physics and technology, vol. 1, no. 1, pp. 2-12, 2008.

[20] R. Kumar and M. Informatics, "Receiver Operating Characteristic (ROC) Curve for Medical Researchers," Indian Pediatrics, vol. 48, no. 4, pp. 277-87, 2011.

[21] W.M. Saridan, "Measurement of Modulation Transfer Function and Wiener Spectrum of Diagnostic X-Ray Screen-Film Systems in a Hospital Setting," (1995).

[22] R.C. Gonzalez, R.E. Woods, and S.L. Eddins, "Digital Image Processing Using Matlab," http://www.lavoisier.fr/notice/frKWOOKASAO3WS6O.html.

[23] O. Marques, Practical Image and Video Processing Using MATLAB. IEEE Press, A John Wiley \& Sons, Inc. International, 2011.

[24] J.M. Pickard, "Using Receiver Operating Characteristic (ROC) Curves to Evaluate Digital Mammography," pp. 1-6, 2004.

[25] N.A. Obuchowski, "Receiver operating characteristic curves and their use in radiology.," Radiology, vol. 229, no. 1, pp. 3-8, 2003.

[26] C.E. Metz, "Basic principles of ROC analysis.," Seminars in nuclear medicine, vol. 8, no. 4, pp. 283-98, 1978.

[27] S.H. Park, J.M. Goo, and C.-H. Jo, "Receiver Operating Characteristic (ROC) Curve: Practical Review for Radiologists," Korean Journal of Radiology, vol. 5, no. 1, pp. 11-8, 2004.

[28] M. Veta, P.J. van Diest, S.M. Willems, et al., "Assessment of algorithms for mitosis detection in breast cancer histopathology images," Medical Image Analysis, vol. 20, no. 1, pp. 237-248, 2015.

[29] J. V. Carter, J. Pan, S.N. Rai, and S. Galandiuk, "ROC-ing along: Evaluation and interpretation of receiver operating characteristic curves," Surgery (United States), vol. 159, no. 6, pp. 1638-45, 2016.

[30] J.A. Hanley and B.J. McNeil, "The meaning and use of the area under a receiver operating characteristic (ROC) curve," Radiology, vol. 143, no. 1, pp. 29-36, 1982. 